\title{
Expression of Bone-related Genes in Bone Marrow MSCs after Cyclic Mechanical Strain: Implications for Distraction Osteogenesis
}

\author{
Meng-chun $\mathrm{Qi}^{1,2}$, Shu-juan Zou ${ }^{1}$, Li-chi Han ${ }^{1}$, Hai-xiao Zhou ${ }^{1}$, Jing Hu ${ }^{1 \text { * }}$ \\ ${ }^{1}$ State Key Laboratory of Oral Diseases, Sichuan University, Chengdu, China \\ ${ }^{2}$ Department of Stomatology, North-China Coal Medical College, Tangshan, China
}

\begin{abstract}
Meng-chun Qi, Shu-juan Zou, Li-chi Han, Hai-xiao Zhou, Jing

Hu. Expression of Bone-related Genes in Bone Marrow MSCs after Cyclic Mechanical Strain: Implications for Distraction Osteogenesis. International Journal of Oral Science, 1(3): 143-150, 2009
\end{abstract}

Aim Understanding the response of mesenchymal stem cells (MSCs) to mechanical strain and their consequent gene expression patterns will broaden our knowledge of the mechanobiology of distraction osteogenesis.

Methodology In this study, a single period of cyclic mechanical stretch $(0.5 \mathrm{~Hz}, 2,000 \mu \varepsilon)$ was performed on rat bone marrow MSCs. Cellular proliferation and alkaline phosphatase (ALP) activity was examined. The mRNA expression of six bone-related genes (Ets-1, bFGF, IGF- II , TGF- $\beta$, Cbfa1 and ALP) was detected using real-time quantitative RT-PCR.

Results The results showed that mechanical strain can promote MSCs proliferation, increase ALP activity, and up-regulate the expression of these genes. A significant increase in Ets-1 expression was detected immediately after mechanical stimulation, but Cbfa1 expression became elevated later. The temporal expression pattern of ALP coincided perfectly with Cbfa1.

Conclusion The results of this study suggest that mechanical strain may act as a stimulator to induce differentiation of MSCs into osteoblasts, and that these bone-related genes may play different roles in the response of MSCs to mechanical stimulation.

Keywords distraction osteogenesis, mechanical strain, mesenchymal stem cell (MSC), osteogenic factor, gene expression
Document code: A

CLC number: Q756
Received Apr. 11, 2009; Revision accepted May 25, 2009

\section{Introduction}

The technique of distraction osteogenesis was applied first in orthopaedic surgery and subsequently is becoming a promising alternative for the treatment of cranio-facial microsoma and bone defects (McCarthy et al., 2001). Bone regeneration during distraction osteogenesis is characterized by a series of cellular processes and complex molecular events that under an appropriate mechanical stimulus result in the differentiation of uncommitted mesenchymal cells into bone-forming cells and eventually the formation of distraction callus (Li et al., 1997). Although the main features of distraction osteogenesis have been reported exten- sively and much knowledge has been acquired, the main cellular and molecular events occurring during distraction osteogenesis have not yet been well characterized.

During distraction osteogenesis, the primary stimulating factor for bone regeneration is mechanical strain created by a distraction device. Determining the molecular events of key cellular components (e.g. mesenchymal stem cells, MSCs) in the callus tissue after each mechanical stimulus will provide valuable information on gene expression patterns, expression sequences and their interactions. Recent studies have indicated that mechanical strain induces osteogenic differentiation of MSCs and that some genes, such as alkaline phosphatase (ALP), 
osteopontin and bone morphogenetic protein (BMP2) are up-regulated in these cells (Sumanasinghe $e t$ al., 2006). However, the response of MSCs to mechanical strain and the consequent dynamic gene expression patterns are still not well understood.

In this study, a four-point bending apparatus was used to perform a single period of cyclic mechanical strain on rat bone marrow MSCs. Expressions of six bone-related genes were determined at different time points after mechanical stimulus using real-time quantitative RT-PCR. These genes included three growth factors: transforming growth factor-beta (TGF- $\beta$ ), basic fibroblast growth factor (bFGF) and insulin-like growth factor- II (IGF- II ); and two transcription factors: core binding factor alpha 1 (Cbfa1/Runx2) and Ets-1, as well as ALP, a well established osteoblast marker. Our hypothesis was that mechanical strain would lead to special expression patterns of these genes and that their expression and their interaction may result in the osteogenic differentiation of MSCs.

\section{Materials and methods}

\section{Harvest and culture of rat bone marrow MSCs}

All procedures regarding the animals used in this study conformed to the guidelines of the Animal Care and Use Committee of Sichuan University. Rat bone marrow MSCs were harvested and cultured using a modification of the method described by Haynesworth et al. (1996). Briefly, bone marrow cells were obtained from femurs and tibia of SD rats and centrifuged at $400 \times \mathrm{g}$ for 10 minutes to remove fat tissue. Cell pellets were suspended in $5 \mathrm{~mL}$ chilled Hanks' balanced salt

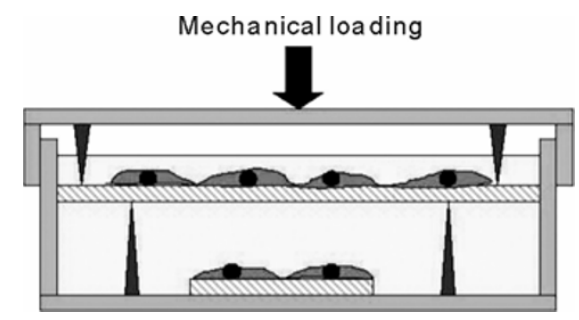

Figure 1 Schematic drawing of apparatus used to apply cyclic uniaxial tensile strain to the cultured cells solution and layered over $8 \mathrm{~mL}$ Percoll solution (1.073 g. $\mathrm{mL}^{-1}$; Phamacia, USA). After centrifugation at $800 \times \mathrm{g}$ for 30 minutes, the mononuclear cell layer was removed from the interface, suspended in $\alpha$-MEM with $15 \%$ fetal bovine serum (FBS), $100 \mathrm{u} \cdot \mathrm{mL}^{-1}$ penicillin, and $100 \mu \mathrm{g} \cdot \mathrm{mL}^{-1}$ streptomycin (Sigma, France) and incubated at $37^{\circ} \mathrm{C}$ with $5 \%$ humid $\mathrm{CO}_{2}$.

\section{Detection of $\mathrm{SH} 3$ by immunocytochemical staining}

To verify that the bone marrow cells obtained were truly pluripotent MSCs, $\mathrm{SH} 3$, a surface antigen marker of MSCs, was detected using immunocytochemical staining. The marrow cells were seeded on coverslips and cultured for 48 hours. The cells were then fixed with $4 \%$ paraformaldehyde for 20 minutes at $4^{\circ} \mathrm{C}$ and washed with phosphatebuffered saline (PBS). Endogenous peroxidase was blocked with $1 \%$ hydrogen peroxide and nonspecific binding was blocked by incubation in PBS containing 1\% blocking reagent (Boehringer Manheim, Canada). For immunostaining, polyclonal goat anti-SH3 antibody (Santa Cruz Biotechnology, USA) was used. Cells were incubated with this antibody (at a dilution of $1: 100$ ) overnight at $4{ }^{\circ} \mathrm{C}$ in a humid chamber. For a negative control, the primary antibody was omitted. A biotinylated antigoat antibody was used as a secondary antibody and the cells were stained using the avidin-biotin complex method. Cell nuclei were counterstained with hematoxylin.

\section{Mechanical stretching of cells}

Cells of passage 3 were seeded onto custommade plastic strips $(8.0 \mathrm{~cm} \times 3.0 \mathrm{~cm})$ at a density of $1.5 \times 10^{5}$ cells/strip and cultured in $150 \mathrm{~cm}^{2}$ stretching dishes for 48 hours. The cells were then subjected to cyclic uniaxial tensile strain $(0.5 \mathrm{~Hz}$, $2,000 \mu \varepsilon)$ using a four-point bending apparatus (Figure 1) described previously (Liu et al., 2006). To exclude the influence of fluid movement on cell gene expression, another strip seeded with cells was placed at the bottom of the stretch device to serve as an experimental control. Cells were subjected to a single period of mechanical stretch for 40 minutes and harvested 0, 0.5, 1, 2, 6, 12 and 
24 hours after mechanical loading. These trials were repeated three times.

\section{Cell proliferation and detection of ALP activity}

The cell number was counted at 24 hours after mechanical loading to assess cell proliferation. To verify osteogenic differentiation of MSCs, ALP histochemical stain (Sigma 85L-3R, USA) was used to detect the presence of ALP activity. The cells sampled at 0 hour and 24 hours after mechanical loading were used for detection.

\section{Detection of gene expression using quantitative real-time RT-PCR}

Quantitative real-time reverse transcription polymerase chain reaction (RT-PCR) using SYBR Green I was performed to detect the expression of six genes, TGF- $\beta$, bFGF, IGF- II, ALP, Cbfa1 and Ets-1. After mechanical loading, total RNA was isolated from the cells and cDNA was prepared using a RevertAid ${ }^{\mathrm{TM}}$ First Strand cDNA Synthesis kit (Femerltas, Lithuania) according to the manufacturer's instructions.

The forward and reverse sequences of the primers in the quantitative PCR are listed in Table 1. $10 \mu \mathrm{L}$ of cDNA sample was added to $200 \mathrm{nmol}$ of each primer, $25 \mu \mathrm{L}$ of $2 \times$ iQ SYBR Green super-mix (Bio-Rad, USA), and PCR-grade water to a volume of $50 \mu \mathrm{L}$. Gene expression was quantified using an ABI Prism 7700 Sequence Detector Real-Time PCR machine (Applied Biosystems, USA). Amplification reactions were conducted with an initial step at $94^{\circ} \mathrm{C}$ for 2 minutes followed by 45 cycles of $53^{\circ} \mathrm{C}$ for 30 seconds and $60^{\circ} \mathrm{C}$ for 40 seconds. Each sample was subjected to tripli- cate PCR reactions and the threshold cycle $(\mathrm{Ct})$ value of samples was normalized by expression of glyceraldehyde-3-phosphate dehydrogenase (GAPDH) as an endogenous house-keeping gene. Standard curves, generated by serial dilutions of $100 \mathrm{ng} \cdot \mu \mathrm{L}^{-1}$ sample cDNA in five 10-fold dilution steps, were used for regression analyses. The subsequent PCR products were also confirmed using agarose gel electrophoresis.

\section{Statistical analysis}

Differences in expression of each gene were analyzed by two-way ANOVA (with time and strain /no strain as the variables) and Tukey post-hoc tests were performed to evaluate the differences between strained cells and the control. One-way ANOVA was also performed to determine if the control cells were affected during mechanical stimulus and if any difference over time was significant. $P \leq 0.05$ was considered statistically significant.

\section{Results}

\section{Culture of bone marrow cells and positive staining for $\mathrm{SH} 3$}

The cells obtained in primary culture proliferated quickly in the first 3 to 4 days and reached confluence within 8 to 12 days. Most of these cells exhibited a fibroblast-like spindle shape in primary culture and changed to polygonal appearance after several passages (Figure 2A). Immunocytochemical staining indicated that these cells were uniformly positive for SH3, a surface marker of MSCs (Figure 2B).

Table 1 Primers used for quantitative real-time RT-PCR reaction

\begin{tabular}{llll}
\hline Accession No. & Gene product & \multicolumn{1}{c}{ Forward primer } & \multicolumn{1}{c}{ Reverse primer } \\
\hline AF153012 & TGF- $\beta$ & CCACACGTCA & CTGGGCAGACAGTTTCGGA \\
X61697 & bFGF & CCACACGTCA & AACACACTTAGAAGCCAGCA \\
X14834 & IGF- II & GCGGCTTCTACTTCAGCA & GGTGGCACAGTATGTCTCCA \\
AF053951 & ALP & CATGTTCCTGGGAGATGGTA & GTGTTGTACGTCTTGGAGAGA \\
AF053951 & Cbfa1 & GCACAGTCCATGCAGTAATATTTA & TGACACGGTGTCACTGCACT \\
L20681 & Ets-1 & GAGTTCAGCCTGAAGGGTGT & CACATCCTCTTTCTGCAGGATCT \\
AF106860 & GAPDH & TGGGTGTGAACCACGAGAA & GGCATGGACTGTGGTCATGA \\
\hline
\end{tabular}




\section{Cell proliferation and ALP histochemical stain}

At 24 hours after mechanical loading, the cell number of the stretched cell group was significantly higher (2.4 fold) than that of the unstretched group (Figure 2C). Histochemical staining showed that ALP-positive cells were detected on all the samples examined. Phase contrast microscopy revealed that $80 \%$ of the cells were ALP-positive cells in the stretched group (Figure 2D), compared with $45 \%$ and $41 \%$ in unstretched and control groups respectively (data not shown).

\section{Detection of growth and transcript factors by RT-PCR}

Transcripts of Ets-1, bFGF, IGF- II , TGF- $\beta$, Cbfa1 and ALP were all detected in rat bone marrow MSCs after mechanical strain but with different mRNA levels and transcript patterns. Agarose gel electrophoresis showed a single product for each gene examined (Figure 3). The standard curves were generated by serial dilutions of $100 \mathrm{ng} \cdot \mu \mathrm{L}^{-1}$ sample cDNA in five 10 -fold dilution steps and excellent linear regression curves were obtained. A representative correlation curve for GAPDH is shown in Figure 3. Statistical analysis showed that the difference in gene expression over time was not significant in control cells.

After mechanical loading, rat bone marrow MSCs showed similar expression patterns for Ets-1 and bFGF but with different mRNA levels (Figures 4A, 4B). Both genes reached maximum transcription at 0.5 hours after mechanical loading and returned to control levels at 6 and 12 hours, respectively. The maximum transcription level increased about 2.66 fold in Ets- 1 and 9.64 fold in bFGF when compared with their corresponding controls. However, the maximum mRNA level of bFGF (123.22\% of GAPDH) was much higher than that of Ets- 1 (27.32\% of GAPDH).

The transcription profiles of IGF- II and TGF- $\beta$ were also similar (Figures 4C, 4D). Both genes reached maximum transcription immediately after mechanical strain and mRNA levels then decreased with time, except for a slight increase at 6 hours. The mRNA of both genes returned to control levels 12 hours after mechanical strain. The maximum transcription level increased about 8.92 fold in

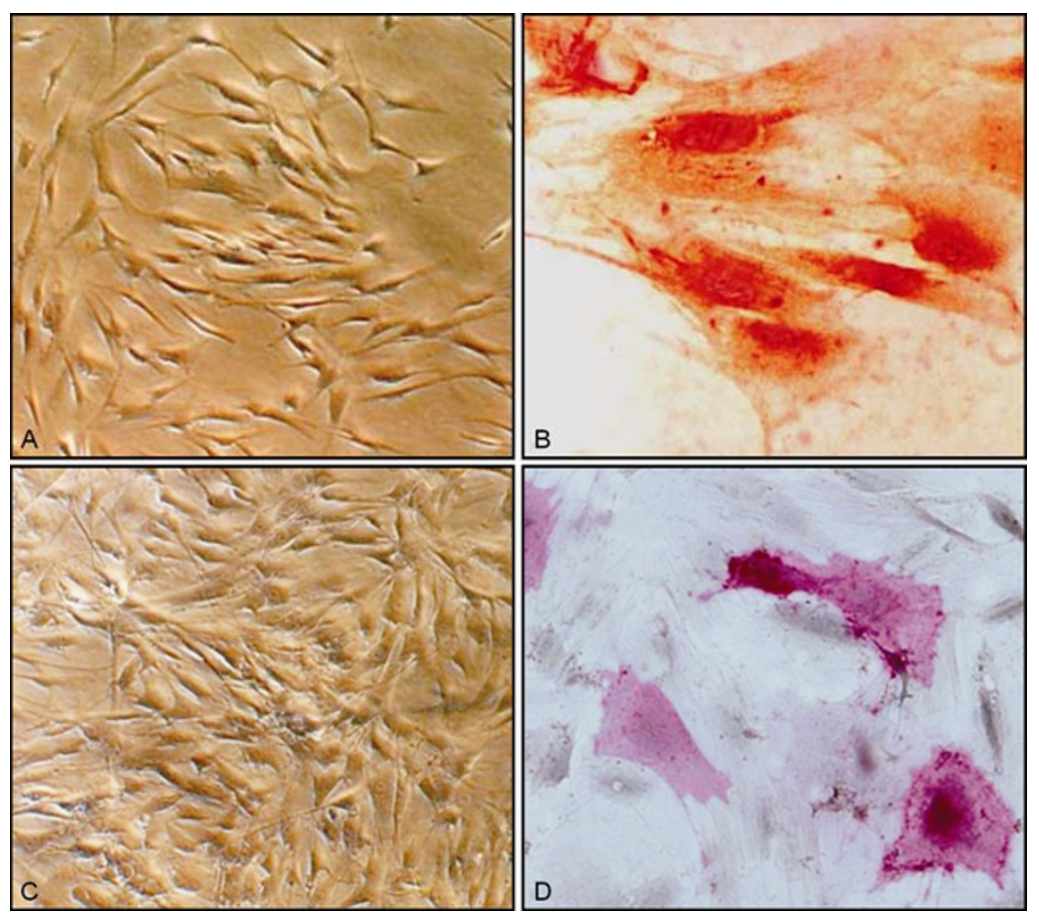

Figure 2 Characterization of MSCs before and after mechanical loading (A): The un-stretched MSCs (original magnification $\times 100$ ). $(\mathrm{B})$ : SH3 positive staining of the cultured cells (original agnification $\times 400$ ).
(C): The stretched cells following 24 hours mechanical strain (original magnification $\times 100$ ). (D): ALP positive staining of the stretched
cells (original magnification $\times 400$ ). 


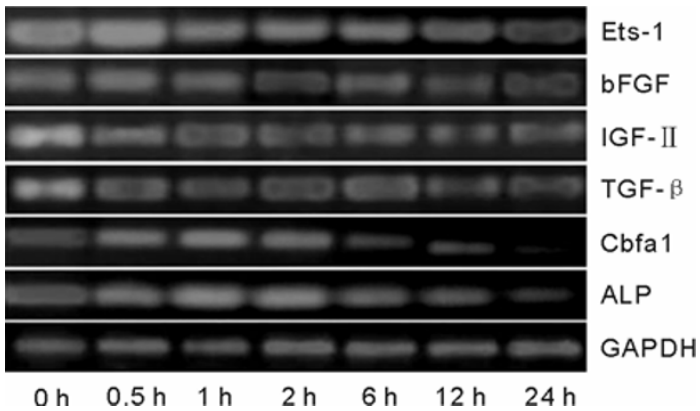

Figure 3 Agarose gel electrophoresis of RT-PCR products for Ets-1, bFGF, IGF- II, TGF- $\beta$, Cbfa1 and ALP at 0, $0.5,1,2,6,12$ and 24 hours after a period of cyclic mechanical strain
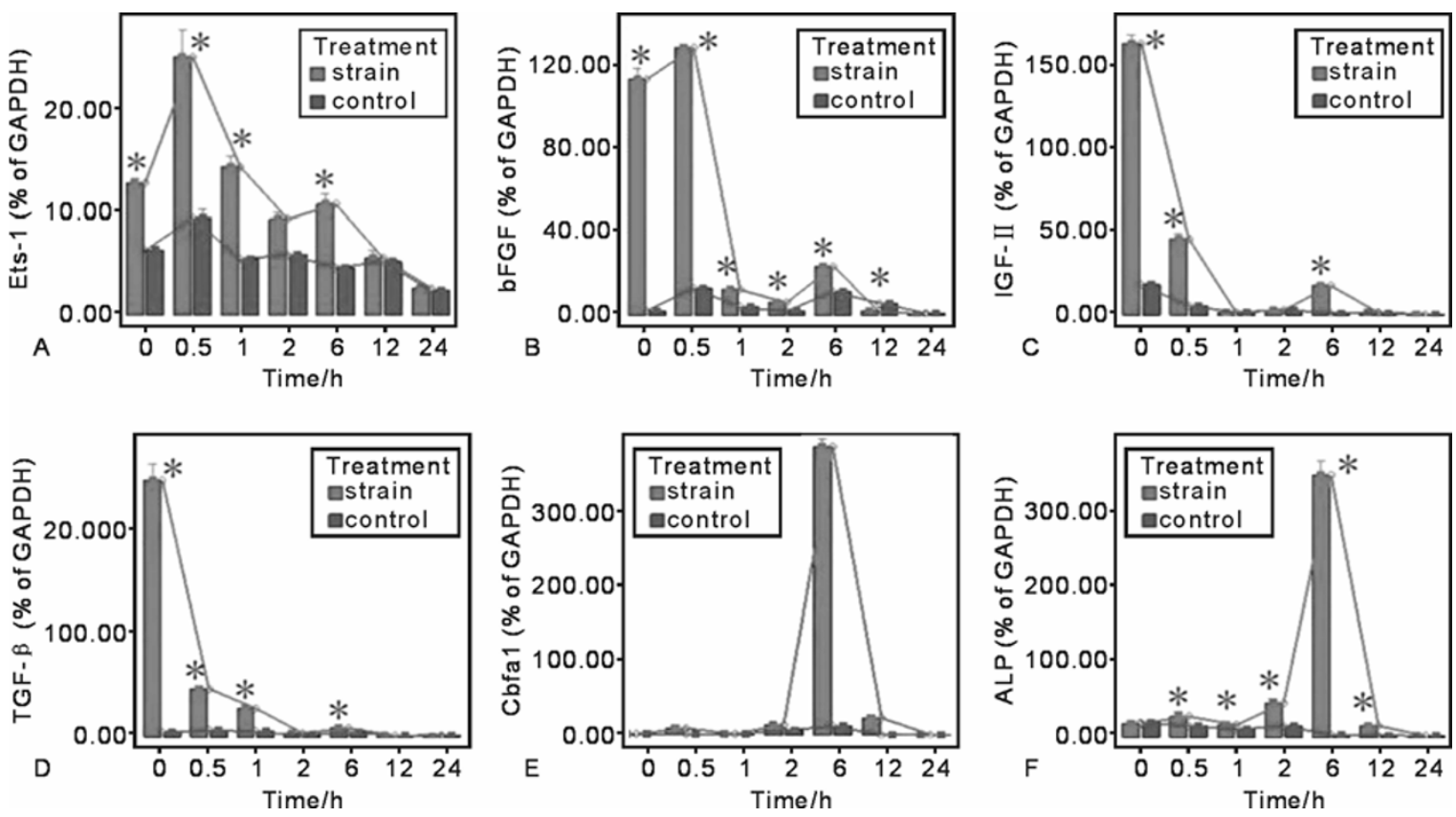

Figure 4 Gene expression of Ets-1(A), bFGF (B), IGF- II (C), TGF- $\beta$ (D), Cbfa1 (E) and ALP (F) in rat bone marrow MSCs at $0,0.5,1,2,6,12$ and 24 hours after a single period of mechanical strain

mRNA levels are expressed as a percentage of GAPDH. Un-stretched control cells were used to exclude the effect of fluid movement on gene expression. ${ }^{*} P<0.05$ or $P<0.01$.

IGF- II and 51.44 fold in TGF- $\beta$ when compared with their corresponding controls. The maximum mRNA level of TGF- $\beta$ ( $265.93 \%$ of GAPDH) was a little higher than that of IGF- II (155.42\% of GAPDH).

Both the expression patterns and mRNA levels of Cbfa1 and ALP were similar (Figures 4E, 4F). After mechanical loading, MSCs exhibited a slow increase in transcription of the two genes within 2 hours and a dramatic increase at 6 hours. Transcription then decreased quickly and returned to control levels 12 hours after mechanical strain.
Maximum transcription increased about 31.30 fold in Cbfa1 and 110.32 fold in ALP when compared with their corresponding controls. The maximum mRNA level of Cbfa1 (372.48\% of GAPDH) was similar to that of ALP (315.51\% of GAPDH).

\section{Discussion}

Differentiation of local uncommitted MSCs into bone-forming cells under mechanical strain and the eventual mineralization of extracellular matrix 
is fundamental to callus formation during distraction osteogenesis. There is no consensus as to the origin of local primitive mesenchymal cells, but bone marrow is one possible source (Yoo et al., 2000). Rat bone marrow cells were used in this study to elucidate the function of MSCs during distraction osteogenesis and their response to mechanical strain. These cells uniformly expressed $\mathrm{SH} 3$, a surface marker of MSCs and underwent osteogeneic, neurogenic and myogenic differentiation under appropriate inducing agents (data not shown). This indicated that the cells used in this study were truly pluripotent MSCs.

Understanding the molecular events within key cellular components (e.g. MSCs) after each mechanical stimulus will facilitate our understanding of the details of the distraction process. In this study, a single period of cyclic mechanical strain $(0.5 \mathrm{~Hz}, 2,000$ microstrain) was applied to rat bone marrow MSCs, and the expression patterns of six bone-related genes were evaluated at different time points after mechanical loading. TGF- $\beta$, bFGF and IGFs are all growth factors that have potent osteogenic potential and play important roles in new bone formation, bone remodeling and fracture healing (Bouletreau et al., 2002). Recent studies (Cillo et al., 2000; Yeung et al., 2001) showed that they make significant contributions to both bone regeneration during distraction osteogenesis and to the response of osteoblasts to mechanical strain. In this study, TGF- $\beta$, bFGF and IGF- II were all actively expressed in MSCs after mechanical strain and significant increases in mRNA transcription were detected at most of the time points when compared with unstretched controls. However, these genes showed different transcription patterns and mRNA levels. The results suggest that these genes may play important roles in regulating the proliferation and differentiation of MSCs and take an active part in determining many consequent cellular characteristics of MSCs caused by mechanical strain. The differences in transcription patterns and mRNA levels may indicate their different roles in these processes.

Cbfa1/Runx2 and Ets-1 are both vital transcription factors that play important roles in regulating the expression of a wide variety of genes responsible for the osteoblast phenotype including osteocalcin, osteopontin, TGF- $\beta$, type I collagen, fibronectin and ALP (Ducy et al., 1997). Recent investigations indicate that they are key regulators of bone development and of differentiation of osteoblasts from their progenitor cells. Mutations in Cbfa1 are responsible for complete blockage of bone formation in mice (Komori et al., 1997). Ets-1 and Cbfa1/Runx2 have also been shown to take an active part in the maturation process of MC3T3-E1 cells, a clonal osteoblastic cell line. Ets-1 plays its role mainly during the first proliferation phase, whereas Cbfa1 contributes mainly to the following differentiation stage. Ets-1 and Cbfa1 can interact with each other to regulate their target genes such as osteopontin (Quarles et al., 1998; Raouf et al., 2000).

The expression patterns of the two transcription factors in mechanically strained MSCs are less well understood. In this study, Ets-1 and Cbfa1 showed clearly different transcription features in mechanically strained MSCs. After mechanical loading, the transcriptional increase of Cbfal first experienced a latency period and then a slight significant increase was observed at 2 hours. Cbfa1 reached its dramatic and maximum transcription at 6 hours after mechanical loading. However, a significant transcriptional increase in Ets-1 was detected immediately after mechanical loading and maximum transcription was observed 0.5 hours later. The difference in transcription between the two genes may indicate their different roles in regulating the response of MSCs to mechanical strain by activating their target genes. Ets-1 may take part in the early response of MSCs to mechanical strain, whereas Cbfa1 may contribute mainly to secondary reactions. Another phenomenon of interest is that the transcriptional pattern of ALP, a target gene for transcription regulation of Cbfa1, coincided perfectly with that of Cbfa1. This similarity verified the close relationship between Cbfa1 and ALP. As ALP is a well established osteoblast phenotypic marker, the temporal expression of this gene indicates the transient differentiation of MSCs into osteoblasts under mechanical strain.

The response of MSCs to mechanical stimulation has been investigated in some previous studies, and their results suggested that appropriate mechanical strain will induce osteogenic differentiation of MSCs and matrix mineralization. Appli- 
cation of equibiaxial cyclic strain $(3 \%, 0.25 \mathrm{~Hz})$ to human MSCs inhibited proliferation but stimulated a 2.3 -fold increase in matrix mineralization over unstrained cells (Simmons et al., 2003). Transcription of some osteoblastic markers, e.g. osteopontin and ALP, was significantly increased in MSCs under cyclic biaxial mechanical strain (1 Hz, 4,000 microstrain). It was also shown that $10 \%$ uniaxial cyclic tensile strain can increase BMP-2 mRNA levels and induce osteogenic differentiation of MSCs (Haynesworth et al., 1996; Sumanasinghe et al., 2006).

Further studies showed that the effect of a mechanical stimulus on MSCs depends on the type and magnitude of the strain. Equiaxial and uniaxial strain can generate different effects on MSCs and, compared with equiaxial strain, uniaxial strain can better promote MSC differentiation into vascular smooth muscle cells and the expression of matrix molecules (Park et al., 2004). A recent study examined the effects of various magnitudes of strain on the ST2 stromal cell line (Koike et al., 2005). The results showed that low levels of strain $(0.8 \%$ and $5 \%)$ increased ALP activity and expression of Runx2. In contrast, high levels of strain decreased ALP activity ( $10 \%$ and $15 \%$ strain) and the expression of osteogenic marker genes (15\% strain). In this study, a low level of cyclic mechanical tensile strain $(0.5 \mathrm{~Hz}, 2,000$ microstrain $)$ was applied to MSCs, and the results showed that, compared with control cells, the expression of all six bone-related genes was up-regulated in strained MSCs. Furthermore, these genes showed different transcript patterns with different mRNA levels, and this may indicate their different roles in regulating the response of MSCs to mechanical strain.

Mechanotransduction pathways during distraction osteogenesis have also been investigated in recent studies. It was shown that an integrinmediated mechanotransduction cascade may participate in the regulation of osseous regeneration up-regulated by mechanical forces during distraction osteogenesis. Activation of initial mechanosensitive components, such as focal adhesion kinase and cellular Src, which is unique to the distraction process, and the increased expression of focal adhesion kinase are closely associated spatially and temporally with the expression of osteogenic precursors, such as bone sialoprotein mRNA (Tong et al., 2003; Rhee et al., 2005). Extracellular signal-related kinase (ERK) 1/2, another important component in mechanotransduction cascades, may be a key signaling convergence point that regulates mechanical force-induced upregulation of MSC osteogenic differentiation and takes part in the process of translocating the osteogenic signal to the nucleus (Xiao et al., 2000). Evidence from recent in vitro and in vivo studies supports the important mechanotransduction function of ERK $1 / 2$ during osteogenic differentiation of MSCs in response to a mechanical stimulus (Rhee et al., 2000).

\section{Conclusion}

In summary, this study demonstrated that a brief tensile strain can promote the proliferation of MSCs, increase their ALP activity and up regulate the expression of two osteogenic transcription factors, Cbfal and Ets-1, as well as bone growth factors such as TGF- $\beta$, bFGF and IGF- II. These findings suggest that mechanical stretching has a direct effect on MSCs, and that mechanical stimulation may be a factor driving osteoblastic differentiation of MSCs during the process of distraction osteogenesis.

\section{Acknowledgements}

This study was supported by grants from the National Nature Science Foundation of China (No. 30772454) and Science and Technology Bureau of Sichuan Province (No. 2006z09-013).

\section{References}

Bouletreau PJ, Warren SM, Longaker MT (2002). The molecular biology of distraction osteogenesis. J CranioMaxillofac Surg, 30(1): 1-11.

Cillo JE, Gassner R, Koepsel RR, Buckley MJ (2000). Growth factor and cytokine gene expression in mechanically strained human osteoblast-like cells: implications for distraction osteogenesis. Oral Surg Oral Med Oral Pathol Oral Radiol Endod, 90(2): 147-154.

Ducy P, Zhang R, Geoffroy V, Ridall AL, Karsenty GG 
(1997). Osf2/Cbfa1: a transcriptional activator of osteoblast differentiation. Cell, 89(5): 747-754.

Haynesworth SE, Baber MA, Caplan AI (1996). Cytokines expression by human marrow-derived mesenchymal progenitor cells in vitro: effects of dexamethasone and IL-1 alpha. J Cell Physiol, 166(3): 585-592.

Koike M, Shimokawa H, Kanno Z, Ohya K, Soma K (2005). Effects of mechanical strain on proliferation and differentiation of bone marrow stromal cell line ST2. J Bone Miner Metab, 23(3): 219-225.

Komori T, Yagi H, Nomura S, Yamaguchi A, Sasaki K, Deguchi K, et al. (1997). Targeted disruption of Cbfal results in a complete lack of bone formation owing to maturational arrests of osteoblasts. Cell, 89(5): 755764.

Li G, Simpson AH, Kenwright J, Triffitt JT (1997). Assessment of cell proliferation in regenerating bone during distraction osteogenesis at different distraction rates. J Orthop Res, 15(5): 765-772.

Liu J, Liu T, Zheng Y, Zhao Z, Liu Y, Cheng H, et al. (2006). Early responses of osteoblast-like cells to different mechanical signals through various signaling pathways. Biochem Biophy Res Commun, 348(3): $1167-1173$.

McCarthy JG, Stelnicki EJ, Mehrara BJ, Longaker MT (2001). Distraction osteogenesis of the craniofacial skeleton. Plast Reconstr Surg, 107(7): 1812-1827.

Park JS, Chu JS, Cheng C, Chen F, Chen D, Li S (2004). Differential effects of equiaxial and uniaxial strain on mesenchymal stem cells. Biotechnol Bioeng, 88(3): 359-368.

Quarles LD, Yohay DA, Lever LW, Caton R, Wenstrup RJ (1992). Distinct proliferative and differentiated stages of murine MC3T3-E1 cells in culture: an in vitro model of osteoblast development. J Bone Miner Res, 7(6): 683-692.

Raouf A, Seth A (2000). Ets transcription factors and targets in osteogenesis. Oncogene, 19(55): 6455-6463.

Rhee ST, Buchman SR (2005). Co-localization of c-Src (pp60src) and bone morphogenetic protein 2/4 expression during mandibular distraction osteogenesis: in vivo evidence of their role within an integrinmediated mechano-transduction pathway. Ann Plast Surg, 55(2): 207-215.

Rhee ST, El-Bassiony L, Buchman SR (2006). Extracellular signal-related kinase and bone morphogenetic protein expression during distraction osteogenesis of the mandible: in vivo evidence of a mechanotransduction mechanism for differentiation and osteogenesis by mesenchymal precursor cells. Plast Reconstr Surg, 117(7): 2243-2249.

Simmons CA, Matlis S, Thornton AJ, Chen S, Wang CY, Mooney DJ (2003). Cyclic strain enhances matrix mineralization by adult human mesenchymal stem cells via the extracellular signal-regulated kinase (ERK 1/2) signaling pathway. J Biomech, 36(8): 1087-1096.

Sumanasinghe RD, Bernacki SH, Loboa EG (2006). Osteogenic differentiation of human mesenchymal stem cells in collagen matrices: effect of uniaxial cyclic tensile strain on bone morphogenetic protein (BMP-2) mRNA expression. Tissue Eng, 12(12): 3459-3465.

Tong L, Buchman SR, Ignelzi MA Jr, Rhee ST, Goldstein SA (2003). Focal adhesion kinase expression during mandibular distraction osteogenesis: evidence for mechanotransduction. Plast Reconst Surg, 111(1): 211222.

Xiao G, Jiang D, Thomas P, Benson MD, Guan K, Karsenty $\mathrm{G}$, et al. (2000). MAPK pathways activate and phosphorylate the osteoblast-specific transcription factor, Cbfa1. J Biol Chem, 275(6): 4453-4459.

Yeung HY, Lee SK, Fung KP, Leung KS (2001). Expression of basic fibroblast growth factor during distraction osteogenesis. Clin Orthop Relat Res, 385: 219-229.

Yoo JU, Johnstone B (1998). The role of osteochondral progenitor cells in fracture repair. Clin Orthop Relat Res, (355 Suppl): S73-81.

\footnotetext{
*Corresponding author: Jing $\mathrm{Hu}$

Address: State Key Laboratory of Oral Diseases, West China College of Stomatology, Sichuan University, No. 14, $3^{\text {rd }}$ Section, Renmin South Road, Chengdu 610041, China

Tel: 862885502334 Fax: 862885582167 E-mail: drhu@vip.sohu.com
} 